\title{
Eigenstructure Assignment by State-derivative and Partial Output-derivative Feedback for Linear Time-invariant Control Systems
}

\author{
T. H. S. Abdelaziz, M. Valášek
}

This paper introduces a parametric approach for solving the problem of eigenstructure assignment via state-derivative feedback for linear control time-invariant systems. This problem is always solvable for any controllable systems if the open-loop system matrix is nonsingular. In this work, the parametric solution to the feedback gain matrix is introduced that describes the available degrees of freedom offered by the state-derivative feedback in selecting the associated eigenvectors from an admissible class. These freedoms can be utilized to improve the robustness of the closed-loop system. Finally, the eigenstructure assignment problem via partial output-derivative feedback is introduced. Numerical examples are included to show the effectiveness of the proposed approach.

Keywords: Eigenstructure assignment, state-derivative feedback, output-derivative feedback, linear systems, feedback stabilization, parametrization.

\section{Introduction}

Eigenstructure assignment is one of the basic techniques for designing linear control systems. The eigenstructure assignment problem is the problem of assigning both a given self-conjugate set of eigenvalues and the corresponding eigenvectors. Assigning the eigenvalues allows one to alter the stability characteristics of the system, while assigning eigenvectors alters the transient response of the system. Eigenstructure assignment via state feedback has developed the design methods for a wide class of linear systems under full-state feedback with the objective of stabilizing control systems. Parametric solutions of eigenstructure assignment for state feedback have been studied by many researchers [4-9].

However, this paper focuses on a special feedback using only state derivatives instead of full-state feedback. Therefore this feedback is called state-derivative feedback. The problem of arbitrary eigenstructure assignment using full-state derivative feedback naturally arises. The motivation for state derivative feedback comes from controlled vibration suppression of mechanical systems. The main sensors of vibration are accelerometers. From accelerations it is possible to reconstruct velocities with reasonable accuracy, but not displacements. Therefore the available signals for feedback are accelerations and velocities only, and these are exactly the derivatives of the states of mechanical systems, which are velocities and displacements. Then, direct measurement of a state is difficult to achieve. One necessary condition for a control strategy to be implementable is that it must use the available measured responses to determine the control action. All of the previous research work in control has assumed that all of the states can be directly measured (i.e., full state feedback).
To control this class of systems, many papers (e.g. [10-15]) have been published describing the acceleration feedback for controlled vibration suppression. However, the eigenstructure assignment approach for feedback gain determination has not been used at all or has not been solved generally. Other papers dealing with acceleration feedback for mechanical systems are [16-17], but here the feedback uses all states (positions, velocities) and accelerations additionally.

Only recently, Abdelaziz and Valášek [1-3] have presented a pole placement technique by state-derivative feedback for single-input and multi-input time-invariant and time-varying linear systems. This paper proposes a parametric approach for eigenstructure assignment in linear systems via state-derivative and partial output-derivative feedback. The necessary and sufficient conditions for the existence of eigenstructure assignment problem are described. The proposed controller is based on measurement and feedback of the state derivatives of the system. This work has successfully extended previous techniques by state feedback and modified to state-derivative feedback. Finally, numerical examples are included to demonstrate the effectiveness of this approach. The main contribution of this work is the efficient technique that solves the eigenstructure assignment problem via state-derivative feedback systems. The procedure defined here represents a unique treatment for the extension of the eigenstructure assignment technique using the derivative feedback in the literature.

The paper is organized as follows. The next section introduces the eigenstructure assignment problem formulation via state-derivative feedback. The necessary and sufficient conditions that ensure solvability are described. Additionally, the parametric solution to the eigenstructure assignment problem via state-derivative feedback for distinct and repeated right eigenvectors is presented. Section 3 introduces the eigenstructure assignment problem by partial output- 
-derivative feedback. Section 4 presents illustrative examples. Finally, conclusions are discussed in section 5 .

\section{Eigenstructure assignment by state-derivative feedback for time-invariant systems}

In this section, we present a parametric approach for solving the eigenstructure assignment problem via state-derivative feedback for linear time-invariant systems.

\subsection{Eigenstructure assignment problem formulation}

Consider a linear, time-invariant, completely controllable system

$$
\dot{\boldsymbol{x}}(t)=\boldsymbol{A} \boldsymbol{x}(t)+\boldsymbol{B} \boldsymbol{u}(t), \quad \boldsymbol{x}\left(t_{0}\right)=\boldsymbol{x}_{0},
$$

where $\boldsymbol{x}(t) \in \mathrm{R}^{n}$ and $\boldsymbol{u}(t) \in \mathrm{R}^{m}$ are the state and the control input vector, respectively, $(m \leq n)$, while $\boldsymbol{A} \in \mathrm{R}^{n \times n}$ and $\boldsymbol{B} \in \mathrm{R}^{n \times m}$ are the system and control gain matrices, respectively. The fundamental assumption imposed on the system is that, the system is completely controllable and the $\boldsymbol{B}$ matrix has a full column rank $m$.

The objective is to stabilize the system by means of a linear feedback that enforces a desired characteristic behavior for the states. The eigenstructure assignment problem is to find the state-derivative feedback control law

$$
\boldsymbol{u}(t)=-\boldsymbol{K} \dot{\boldsymbol{x}}(t)
$$

which assigns the prescribed closed-loop eigenvalues and the corresponding eigenvectors that stabilize the system and achieve the desired performance. Then, the closed-loop system dynamics becomes:

$$
\dot{\boldsymbol{x}}(t)=\left(\boldsymbol{I}_{n}+\boldsymbol{B} \boldsymbol{K}\right)^{-1} \boldsymbol{A} \boldsymbol{x}(t),
$$

where $\boldsymbol{I}_{n} \in R^{n \times n}$ is the identity matrix. In what follows, we assume that $\left(\boldsymbol{I}_{n}+\boldsymbol{B} \boldsymbol{K}\right)$ has a full rank in order that the closed-loop system is well define. The closed-loop characteristic polynomial is given by

$$
\operatorname{det}\left[\lambda \boldsymbol{I}_{n}-\left(\boldsymbol{I}_{n}+\boldsymbol{B} \boldsymbol{K}\right)^{-1} \boldsymbol{A}\right]=0
$$

Denote the right eigenvector matrix of the closed-loop matrix $\left(\boldsymbol{I}_{n}+\boldsymbol{B} \boldsymbol{K}\right)^{-1} \boldsymbol{A}$ by $\boldsymbol{V}$, and we then have by definition,

$$
\left(\boldsymbol{I}_{n}+\boldsymbol{B K}\right)^{-1} \boldsymbol{A} \boldsymbol{V}=\boldsymbol{V} \boldsymbol{\Lambda}
$$

where $\boldsymbol{\Lambda}=\operatorname{diag}\left\{\lambda_{1}, \ldots, \lambda_{n}\right\}$ and $\operatorname{det}(\boldsymbol{V}) \neq 0$.

We now formulate the eigenstructure assignment problem via state-derivative feedback as follows.

Eigenstructure Assignment Problem 1: Given the controllable pair $(\boldsymbol{A}, \boldsymbol{B})$ and the desired self-conjugate set $\left\{\lambda_{1}, \ldots, \lambda_{n}\right\} \in \mathrm{C}$. Select the appropriate state-derivative feedback gain matrix $\boldsymbol{K} \in \mathrm{R}^{n \times m}$ that will make the closed-loop matrix $\left(\boldsymbol{I}_{n}+\boldsymbol{B} \boldsymbol{K}\right)^{-1} \boldsymbol{A}$ have admissible eigenvalues and associated set of eigenvectors $\boldsymbol{V}$.

Now, we will prove the necessary and sufficient conditions for the existence of the eigenstructure assignment problem via state-derivative feedback. The necessary conditions to ensure solvability are presented in the following theorem.

\section{Theorem 1:}

The eigenstructure assignment problem for the real pair $(\boldsymbol{A}, \boldsymbol{B})$ is solvable for any arbitrary self-conjugate closed-loop poles, if and only if $(\boldsymbol{A}, \boldsymbol{B})$ is completely controllable, that is,

$$
\begin{gathered}
\operatorname{rank}\left[\boldsymbol{B}, \boldsymbol{A B}, \ldots, \boldsymbol{A}^{n-1} \boldsymbol{B}\right]=n \\
\text { and } \\
\operatorname{rank}\left[\lambda \boldsymbol{I}_{n}-\boldsymbol{A}, \boldsymbol{B}\right]=n, \forall \lambda \in \mathrm{C},
\end{gathered}
$$

and matrix $\boldsymbol{A}$ is nonsingular.

Proof: From the condition of that the closed-loop matrix must be defined. This means the matrix $\left(\boldsymbol{I}_{n}+\boldsymbol{B K}\right)$ is of full rank and $\operatorname{det}\left(\boldsymbol{I}_{n}+\boldsymbol{B} \boldsymbol{K}\right) \neq 0$. Then, from (5) it is easy to rewrite as

$$
A \boldsymbol{V}-\boldsymbol{V} \boldsymbol{\Lambda}=\boldsymbol{B} \boldsymbol{K} \boldsymbol{\Lambda} \boldsymbol{\Lambda}
$$

which can be written as

$$
\boldsymbol{B} \boldsymbol{K}=\boldsymbol{A} \boldsymbol{V} \boldsymbol{\Lambda}^{-1} \boldsymbol{V}^{-1}-\boldsymbol{I}_{n}
$$

Then,

$$
\begin{aligned}
\operatorname{det}\left(\boldsymbol{I}_{n}+\boldsymbol{B} \boldsymbol{K}\right) & =\operatorname{det}\left(\boldsymbol{I}_{n}+\boldsymbol{A} \boldsymbol{V} \boldsymbol{\Lambda}^{-1} \boldsymbol{V}^{-1}-\boldsymbol{I}_{n}\right)= \\
& =\operatorname{det}\left(\boldsymbol{A} \boldsymbol{V} \boldsymbol{\Lambda}^{-1} \boldsymbol{V}^{-1}\right) \neq 0 .
\end{aligned}
$$

Since $\boldsymbol{V}$ and $\boldsymbol{\Lambda}$ must be nonsingular. This leads to, $\operatorname{det}(\boldsymbol{A}) \neq 0$, and matrix $\boldsymbol{A}$ must be of full rank in order for the matrix to be defined.

Thus, the necessary and sufficient conditions for the existence of the solution to the eigenstructure assignment problem via state-derivative feedback is that the system is completely controllable and all eigenvalues of the original system are nonzero ( $\boldsymbol{A}$ has full rank). We remark that the requirement that the matrix $\boldsymbol{\Lambda}$ is diagonal, together with the invertibility of $V$, ensures that the closed-loop system is non-defective.

Based on the above necessary and sufficient conditions, the parametric formula for the state-derivative feedback gain matrix $\boldsymbol{K}$ that assigns the desired closed-loop poles is derived.

\subsection{Case of distinct eigenvalues}

In this subsection, all the desired closed-loop eigenvalues $\left\{\lambda_{1}, \ldots, \lambda_{n}\right\}$ are distinct.

Let $\left(\lambda_{i}, \mathbf{v}_{\mathbf{i}}\right)$ be the closed-loop eigenvalue and the corresponding eigenvector, then we can write the right eigenvectors of the closed-loop system as

$$
\left(\boldsymbol{I}_{n}+\boldsymbol{B} \boldsymbol{K}\right)^{-} \boldsymbol{A} \mathbf{v}_{i}=\lambda_{i} \mathbf{v}_{i}, \quad i=1, \ldots, n .
$$

The above equation can be rewritten as

$$
\boldsymbol{A} \mathbf{v}_{i}=\lambda_{i}\left(\boldsymbol{I}_{n}+\boldsymbol{B} \boldsymbol{K}\right) \mathbf{v}_{i}
$$

then

$$
A \mathbf{v}_{i}-\lambda_{i} \mathbf{v}_{i}=\lambda_{i} B \boldsymbol{K} \mathbf{v}_{i} .
$$

Let $\mathbf{w}_{i}=\boldsymbol{K} \mathbf{v}_{i}$, then we have

$$
A \mathbf{v}_{i}-\lambda_{i} \mathbf{v}_{i}=\lambda_{i} B \mathbf{w}_{i} .
$$

Then (12) can be rewritten in a matrix form as,

$$
\left[\lambda_{i} \boldsymbol{I}_{n}-\boldsymbol{A}, \lambda_{i} \boldsymbol{B}\right]\left(\begin{array}{c}
\mathbf{v}_{i} \\
\mathbf{w}_{i}
\end{array}\right)=\mathbf{0}, \quad i=1, \ldots, n .
$$

Thus the subspace may be calculated in which the closed-loop eigenvector, $\mathbf{v}_{i}$, and gain-eigenvector product, $\mathbf{w}_{i}$, must satisfy the null space, null $\left(\left[\lambda_{i} \boldsymbol{I}_{n}-\boldsymbol{A}, \lambda_{i} \boldsymbol{B}\right]\right), i=1, \ldots, n$. 
Finally, the parametric equation to the right eigenvector can be expressed as

$$
\left[\lambda_{i} \boldsymbol{I}_{n}-\boldsymbol{A}, \lambda_{i} \boldsymbol{B}\right] \boldsymbol{\xi}_{i}=\mathbf{0}, \quad \boldsymbol{\xi}_{i}=\left(\begin{array}{c}
\mathbf{v}_{i} \\
\boldsymbol{K} \mathbf{v}_{i}
\end{array}\right)=\left(\begin{array}{c}
\mathbf{v}_{i} \\
\mathbf{w}_{i}
\end{array}\right) .
$$

Then $\mathbf{v}_{i} \in \mathrm{C}^{n}$ and $\boldsymbol{K} \in \mathrm{R}^{m \times n}$ are unknown and notational simplicity is gained by defining the $(n+m) \times 1$ vector $\boldsymbol{\xi}_{i}$. The determination of $\boldsymbol{K}$ consists of two general steps. First, a sufficient number of solution vectors $\xi_{i}$ is found. Then, the internal structure among the components of these vectors is used to find the feedback gain matrix $\boldsymbol{K}$.

Then, there exists a real feedback gain matrix $\boldsymbol{K}$ if and only if the following three conditions are satisfied:

1. The assigned eigenvalues are symmetric with respect to the real axis.

2. The vectors $\left\{\mathbf{v}_{i} \in \mathrm{C}^{n}, i=1, \ldots, n\right\}$, are linearly independent and for complex-conjugate poles, $\lambda_{i}=\bar{\lambda}_{j}$ then $\mathbf{v}_{i}=\overline{\mathbf{v}}_{j}$.

3. There exists a set of vectors $\left\{\mathbf{w}_{i} \in \mathrm{C}^{m}, i=1, \ldots, n\right\}$ satisfying (13) and $\mathbf{w}_{i}=\overline{\mathbf{w}}_{j}$ for $\lambda_{i}=\bar{\lambda}_{j}$.

The parametric formula for the state-derivative feedback gain matrix $\boldsymbol{K}$ that assigns the desired closed-loop eigenvalues and eigenvectors is derived as

$$
\boldsymbol{K}\left[\mathbf{v}\left(\lambda_{1}\right), \ldots, \mathbf{v}\left(\lambda_{n}\right)\right]=\left[\mathbf{w}\left(\lambda_{1}\right), \ldots, \mathbf{w}\left(\lambda_{n}\right)\right]
$$

Finally the gain matrix can be computed as

$$
\boldsymbol{K}=\boldsymbol{W} \boldsymbol{V}^{-1}
$$

where

$$
\boldsymbol{V}=\left[\mathbf{v}\left(\lambda_{1}\right), \ldots, \mathbf{v}\left(\lambda_{n}\right)\right] \text { and } \boldsymbol{W}=\left[\mathbf{w}\left(\lambda_{1}\right), \ldots, \mathbf{w}\left(\lambda_{n}\right)\right] .
$$

This is a parametric solution to the eigenstructure assignment problem via state-derivative feedback. It is clear that, for the case of multi-input, there are infinite solutions to the feedback gain. Eigenstructure assignment uses the extra degrees of freedom in the undetermined solution to specify the closed-loop right-eigenvectors, $\boldsymbol{V}$, corresponding to the desired self-conjugate set $\left\{\lambda_{i}\right\}, i=1, \ldots, n$.

\subsection{Case of repeated eigenvalues}

Our main work is to find a parametric solution in the case that some or all of the desired closed-loop eigenvalues $\left\{\lambda_{i}\right\}$ are repeated.

Let $\Gamma=\left\{\lambda_{i}, \lambda_{i} \in \mathrm{C}, i=1, \ldots, s, 1 \leq s<n\right\}$ be a set of desired eigenvalues and let us denote the algebraic and geometric multiplicity of $\lambda_{i}$ by $m_{i}$ and $q_{i}$, respectively, $\left(q_{i} \leq m_{i}\right)$. The length of $q_{i}$ chains of generalized eigenvectors with $\lambda_{i}$ is denoted by $p_{i j},\left(j=1, \ldots, q_{i}\right)$. It is satisfying that $\sum_{i=1}^{s} \sum_{j=1}^{q_{i}} p_{i j}=n$.

Then, the right generalized eigenvector of the closed-loop system with $\lambda_{i}$ is denoted by $\mathbf{v}_{i j}^{k} \in \mathrm{C}^{n}, i=1, \ldots, s$, $j=1, \ldots, q_{i}, k=1, \ldots, p_{i j}$. Then it is satisfied that

$$
\begin{aligned}
& \left(\boldsymbol{I}_{n}+\boldsymbol{B} \boldsymbol{K}\right)^{-1} \boldsymbol{A} \mathbf{v}_{i j}^{k}=\lambda_{i} \mathbf{v}_{i j}^{k}+\mathbf{v}_{i j}^{k-1}, \quad \mathbf{v}_{i j}^{0}=0, \\
& i=1, \ldots, s, \quad j=1, \ldots, q_{i}, \quad k=1, \ldots, p_{i j} .
\end{aligned}
$$

This equation demonstrates the relation of assignable right generalized eigenvectors with the corresponding eigenvalue, and can be rewritten as,
$\boldsymbol{A} \mathbf{v}_{i j}^{k}=\lambda_{i}\left(\boldsymbol{I}_{n}+\boldsymbol{B} \boldsymbol{K}\right) \mathbf{v}_{i j}^{k}+\left(\boldsymbol{I}_{n}+\boldsymbol{B} \boldsymbol{K}\right) \mathbf{v}_{i j}^{k-1}, \quad \mathbf{v}_{i j}^{0}=0$.

Let $\mathbf{w}_{i j}^{k}=\boldsymbol{K} \mathbf{v}_{i j}^{k}, i=1, \ldots, s, j=1, \ldots, q_{i}, k=1, \ldots, p_{i j}$. The notations are defined as $\boldsymbol{V}=\left[\boldsymbol{V}_{1}, \ldots, \boldsymbol{V}_{s}\right], \boldsymbol{V}_{i}=\left[\boldsymbol{V}_{i 1}, \ldots, \boldsymbol{V}_{i q_{i}}\right]$, $\boldsymbol{V}_{i j}=\left[\mathbf{v}_{i j}^{1}, \ldots, \mathbf{v}_{i j}^{p_{i j}}\right]$. The set of $\mathbf{w}_{i j}^{k}$ is define in a similar manner to the set of $\mathbf{v}_{i j}^{k}$. This leads to

$\left[\lambda_{i} \boldsymbol{I}_{n}-\boldsymbol{A}, \lambda_{i} \boldsymbol{B}\right]\left(\begin{array}{c}\mathbf{v}_{i j}^{k} \\ \mathbf{w}_{i j}^{k}\end{array}\right)=-\left[\boldsymbol{I}_{n}, \boldsymbol{B}\right]\left(\begin{array}{c}\mathbf{v}_{i j}^{k-1} \\ \mathbf{w}_{i j}^{k-1}\end{array}\right), \mathbf{v}_{i j}^{0}=\boldsymbol{0}, \mathbf{w}_{i j}^{0}=\boldsymbol{0}$.

Finally, the parametric equation to the right generalized eigenvector can be expressed as

$\left[\lambda_{i} \boldsymbol{I}_{n}-\boldsymbol{A}, \lambda_{i} \boldsymbol{B}\right] \boldsymbol{\xi}_{i j}^{k}=-\left[\boldsymbol{I}_{n}, \boldsymbol{B}\right] \boldsymbol{\xi}_{i j}^{k-1}, \boldsymbol{\xi}_{i j}^{k}=\left(\begin{array}{c}\mathbf{v}_{i j}^{k} \\ \mathbf{w}_{i j}^{k}\end{array}\right), \boldsymbol{\xi}_{i j}^{0}=\mathbf{0}$,

$i=1, \ldots, s, j=1, \ldots, q_{i}, k=1, \ldots, p_{i j}$.

Similar to the case of distinct eigenvalues the $(n+m)$-dimentional parameter vectors $\boldsymbol{\xi}_{i j}^{k}$ are chosen arbitrarily, under the condition that the columns of eigenvector matrix $\boldsymbol{V}$ are linearly independent.

A parametric solution $K$ to the eigenstructure assignment problem via state-derivative feedback is given by

$$
\boldsymbol{K}=\boldsymbol{W} \boldsymbol{V}^{-}
$$

where

$$
\boldsymbol{V}=\left[V_{1}\left(\lambda_{1}\right), \ldots, V_{s}\left(\lambda_{s}\right)\right] \text { and } \boldsymbol{W}=\left[W_{1}\left(\lambda_{1}\right), \ldots, W_{s}\left(\lambda_{s}\right)\right] .
$$

Then the feedback gain matrix is parameterized directly in terms of the eigenstructure of the closed-loop system, which can be selected to ensure robustness by exploiting the freedom of these parameters.

Then, there exists a real feedback gain matrix $\boldsymbol{K}$, such that (19) holds, if and only if the following three conditions are satisfied:

1. The assigned eigenvalues are symmetric with respect to the real axis.

2. The vectors

$$
\left\{\mathbf{v}_{i j}^{k} \in \mathrm{C}^{n}, i=1, \ldots, s, j=1, \ldots, q_{i}, k=1, \ldots, p_{i j}\right\} \text { are }
$$

linearly independent and for complex-conjugate poles, $\lambda_{i_{1}}=\bar{\lambda}_{i_{2}}$, then $\mathbf{v}_{i_{1} j}^{k}=\overline{\mathbf{v}}_{i_{2} j}^{k}$.

3. There exists a set of vectors $\left\{\mathbf{w}_{i j}^{k} \in \mathrm{C}^{m}, i=1, \ldots, s, j=1, \ldots, q_{i}, k=1, \ldots, p_{i j}\right\}$, satisfying (19) and $\mathbf{w}_{i_{1} j}^{k}=\overline{\mathbf{w}}_{i_{2} j}^{k}$ for $\lambda_{i_{1}}=\bar{\lambda}_{i_{2}}$.

From the above results we observe that the system poles can always be assigned by a state-derivative feedback for any controllable system if and only if all eigenvalues of the original system are nonzero. In the case of single-input, $m=1$, there is only at most one solution. In the case of multi-input, $m>1$, the solution is generally non-unique, and extra conditions must be imposed to specify a solution. The extra freedom can be used to give the closed-loop system other desirable properties. The extra freedom can be used in different ways, for example to decrease the norm of the feedback gain matrix or to improve the condition of the eigenvalues of the closed-loop matrix. Additionally, the robustness of the closed-loop system against system parameter 
perturbation is increased. This issue becomes very important when the system model is not sufficently precise or the system is subject to parameter uncertainty.

\section{Eigenstructure assignment by partial output-derivative feedback for time-invariant systems}

Let us consider eigenstructure assignment via output-derivative feedback for linear time-invariant systems. In practice, it may be expensive to measure all the state-derivative variables, or they may not all be available for measurement. We then feedback some of the derivative-outputs via a controller. Output-derivative feedback is a more difficult problem than state-derivative feedback.

Consider a linear, time-invariant, completely controllable and observable system

$$
\begin{aligned}
\dot{\boldsymbol{x}}(t) & =\boldsymbol{A} \boldsymbol{x}(t)+\boldsymbol{B} \boldsymbol{u}(t), \quad \boldsymbol{x}\left(t_{0}\right)=\boldsymbol{x}_{0}, \\
\boldsymbol{y}(t) & =\boldsymbol{C} \boldsymbol{x}(t),
\end{aligned}
$$

where the state $x(t) \in \mathrm{R}^{n}$, the output $\boldsymbol{y}(t) \in \mathrm{R}^{r}$ and the control input vector $\boldsymbol{u}(t) \in \mathrm{R}^{m}$, while $\boldsymbol{A} \in \mathrm{R}^{n \times n}, \boldsymbol{B} \in \mathrm{R}^{n \times m}$ and $\boldsymbol{C} \in \mathrm{R}^{r \times n}$ are the system, control and output gain matrices, respectively. The fundamental assumptions imposed on the system are that the system is completely controllable and observable. Additionally, matrices $\boldsymbol{B}$ and $\boldsymbol{C}$ have a full rank.

The objective is to stabilize the system by means of a linear feedback that enforces a desired characteristic behavior for the state. The eigenstructure assignment problem is to find the output-derivative feedback control law

$$
\boldsymbol{u}(t)=-\boldsymbol{F} \dot{\boldsymbol{y}}(t),
$$

which assigns prescribed closed-loop eigenvalues that stabilize the system and achieve the desired performance, and the closed-loop system dynamics becomes

$$
\dot{\boldsymbol{x}}(t)=\left(\boldsymbol{I}_{n}+\boldsymbol{B} \boldsymbol{F C}\right) \boldsymbol{A} \boldsymbol{x}(t)
$$

In what follows, we assume that $\left(\boldsymbol{I}_{n}+\boldsymbol{B} \boldsymbol{F C}\right)$ has a full rank in order that the closed-loop system is well defined. The closed-loop characteristic equation of this system is given by

$$
\operatorname{det}\left[\lambda \boldsymbol{I}_{n}-\left(\boldsymbol{I}_{n}+\boldsymbol{B} \boldsymbol{F} \boldsymbol{C}\right)^{-1} \boldsymbol{A}\right]=0
$$

Denote the right eigenvector matrix of the matrix $\left(\boldsymbol{I}_{n}+\boldsymbol{B} \boldsymbol{F} \boldsymbol{C}\right)^{-1} \boldsymbol{A}$ by $\boldsymbol{V}$, and then we have,

$$
\left(\boldsymbol{I}_{n}+\boldsymbol{B F C}\right)^{-1} \boldsymbol{A} \boldsymbol{V}=\boldsymbol{V} \boldsymbol{\Lambda} .
$$

Now, the eigenstructure assignment problem for output-derivative feedback is as follows.

Eigenstructure Assignment Problem 2: Given the reat triple $(\boldsymbol{A}, \boldsymbol{B}, \boldsymbol{C})$ and the desired self-conjugate set $\left\{\lambda_{1}, \ldots, \lambda_{n}\right\} \in \mathrm{C}$. Select the appropriate output-derivative feedback matrix $\boldsymbol{F} \in \mathrm{R}^{m \times r}$ that will make the closed-loop matrix $\left(\boldsymbol{I}_{n}+\boldsymbol{B} \boldsymbol{F C}\right)^{-1} \boldsymbol{A}$ have admissable eigenvalues and corresponding right generalized eigenvectors $\boldsymbol{V}$.

Now, the necessary and sufficient conditions for the existence of the eigenstructure assignment problem via output-derivative feedback are presented in the following theorem.

\section{Theorem 2:}

The eigenstructure assignment problem for the triple $(\boldsymbol{A}, \boldsymbol{B}, \boldsymbol{C})$ is solvable for any arbitrary self-conjugate closed-loop poles, if and only if $(\boldsymbol{A}, \boldsymbol{B})$ is completely controllable, that is,

$$
\begin{aligned}
& \operatorname{rank}\left[\boldsymbol{B}, \boldsymbol{A} \boldsymbol{B}, \ldots, \boldsymbol{A}^{n-1} \boldsymbol{B}\right]=n \text { and } \\
& \operatorname{rank}\left[\lambda \boldsymbol{I}_{n}-\boldsymbol{A}, \boldsymbol{B}\right]=n, \forall \lambda \in \mathrm{C},
\end{aligned}
$$

also, the pair $(\boldsymbol{A}, \boldsymbol{C})$ is completely observable that is,

$$
\begin{aligned}
& \operatorname{rank}\left[\boldsymbol{C}, \boldsymbol{A} \boldsymbol{C}, \ldots, \boldsymbol{A}^{n-1} \boldsymbol{C}\right]=n \text { and } \\
& \operatorname{rank}\left[\lambda \boldsymbol{I}_{n}-\boldsymbol{A}, \boldsymbol{C}\right]=n, \forall \lambda \in \mathrm{C},
\end{aligned}
$$

and matrix $\boldsymbol{A}$ is nonsingular.

Proof: From the condition of that the closed-loop matrix must be defined. This means the matrix $\left(\boldsymbol{I}_{n}+\boldsymbol{B F C}\right)$ is of full rank and $\operatorname{det}\left(\boldsymbol{I}_{n}+\boldsymbol{B} \boldsymbol{F C}\right) \neq 0$. Then, from (26) it can easily put in the following form

$$
A V-V \Lambda=B F C V \Lambda
$$

which can be written as

$$
\boldsymbol{B} \boldsymbol{F} \boldsymbol{C}=\boldsymbol{A} \boldsymbol{V} \boldsymbol{\Lambda}^{-1} \boldsymbol{V}^{-1}-\boldsymbol{I}_{n}
$$

Then,

$$
\begin{aligned}
\operatorname{det}\left[\boldsymbol{I}_{n}+\boldsymbol{B} \boldsymbol{F} \boldsymbol{C}\right] & =\operatorname{det}\left[\boldsymbol{I}_{n}+\boldsymbol{A} \boldsymbol{V} \boldsymbol{\Lambda}^{-1} \boldsymbol{V}^{-1}-\boldsymbol{I}_{n}\right]= \\
& =\operatorname{det}\left[\boldsymbol{A} \boldsymbol{V} \boldsymbol{\Lambda}^{-1} \boldsymbol{V}^{-1}\right] \neq 0
\end{aligned}
$$

Since $\boldsymbol{V}$ and $\boldsymbol{\Lambda}$ must be nonsingular. Thus, matrix $\boldsymbol{A}$ must be of full rank in order for the closed-loop matrix to be defined.

From the above results we can observe that, the necessary and sufficient conditions for existence the solution to the eigenstructure assignment problem via output-derivative feedback are the system is completely controllable and observable and all eigenvalues of the original system are nonzero ( $\boldsymbol{A}$ has full rank).

Our main work in this section is to find a parametric solution to the gain matrix in the case of right generalized eigenvectors.

Let $\Gamma=\left\{\lambda_{i}, \lambda_{i} \in \mathrm{C}, \quad i=1, \ldots, s, \quad 1 \leq s<n\right\}$ be a set of desired eigenvalues and let us denote the algebraic and geometric multiplicity of $\lambda_{i}$ by $m_{i}$ and $q_{i}$, respectively. The length of $q_{i}$ chains of generalized eigenvectors with $\lambda_{i}$, are denoted by $p_{i j}$. The right generalized eigenvector of the closed-loop system with $\lambda_{i}$ is denoted by $\mathbf{v}_{i j}^{k} \in \mathrm{C}^{n}, i=1, \ldots, s, j=1, \ldots, q_{i}$, $k=1, \ldots, p_{i j}$. It is satisfying that $\sum_{i=1}^{s} \sum_{j=1}^{q_{i}} p_{i j}=n$. Then we have the following relation

$$
\begin{aligned}
& \left(\boldsymbol{I}_{n}+\boldsymbol{B} \boldsymbol{F C}\right)^{-1} \boldsymbol{A} \mathbf{v}_{i j}^{k}=\lambda_{i} \mathbf{v}_{i j}^{k}+\mathbf{v}_{i j}^{k-1}, \quad \mathbf{v}_{i j}^{0}=\mathbf{0}, \\
& i=1, \ldots, s, \quad j=1, \ldots, q_{i}, \quad k=1, \ldots, p_{i j} .
\end{aligned}
$$

This equation demonstrates the relation of assignable right generalized eigenvectors with the eigenvalue, and can be rewritten,

$$
\begin{gathered}
\left(\boldsymbol{A}-\lambda_{i} \boldsymbol{I}_{n}+\lambda_{i} \boldsymbol{B F C}\right) \mathbf{v}_{i j}^{k}=\left(\boldsymbol{I}_{n}+\boldsymbol{B} \boldsymbol{F C}\right) \mathbf{v}_{i j}^{k-1}, \quad \mathbf{v}_{i j}^{0}=\mathbf{0} . \\
\text { Let } \mathbf{z}_{i j}^{k}=\boldsymbol{F} \boldsymbol{C} \mathbf{v}_{i j}^{k}, i=1, \ldots, s, j=1, \ldots, q_{i}, k=1, \ldots, p_{i j} \text {. The } \\
\text { notations are defined as } \boldsymbol{V}=\left[\boldsymbol{V}_{1}, \ldots, \boldsymbol{V}_{s}\right], \boldsymbol{V}_{i}=\left[\boldsymbol{V}_{i 1}, \ldots, \boldsymbol{V}_{i q_{i}}\right],
\end{gathered}
$$


$\boldsymbol{V}_{i j}=\left[\mathbf{v}_{i j}^{1}, \ldots, \mathbf{v}_{i j}^{p_{i j}}\right]$. The set of $\mathbf{z}_{i j}^{k}$ is defined in a similar manner to the set of $\mathbf{v}_{i j}^{k}$. The above equation can be rewritten as $\left[\lambda_{i} \boldsymbol{I}_{n}-\boldsymbol{A}, \lambda_{i} \boldsymbol{B}\right]\left(\begin{array}{c}\mathbf{v}_{i j}^{k} \\ \mathbf{z}_{i j}^{k}\end{array}\right)=-\left[\boldsymbol{I}_{n}, \boldsymbol{B}\right]\left(\begin{array}{c}\mathbf{v}_{i j}^{k-1} \\ \mathbf{z}_{i j}^{k-1}\end{array}\right), \mathbf{v}_{i j}^{0}=\boldsymbol{0}, \mathbf{z}_{i j}^{0}=\mathbf{0}$.

The right generalized eigenvector can be expressed as

$$
\left[\lambda_{i} \boldsymbol{I}_{n}-\boldsymbol{A}, \lambda_{i} \boldsymbol{B}\right] \boldsymbol{\psi}_{i j}^{k}=-\left[\boldsymbol{I}_{n}, \boldsymbol{B}\right] \boldsymbol{\psi}_{i j}^{k-1}, \boldsymbol{\psi}_{i j}^{k}=\left(\begin{array}{c}
\mathbf{v}_{i j}^{k} \\
\mathbf{z}_{i j}^{k}
\end{array}\right), \boldsymbol{\psi}_{i j}^{0}=\boldsymbol{0},
$$

$i=1, \ldots, s, j=1, \ldots, q_{i}, \quad k=1, \ldots, p_{i j}$.

Similar to the case of state-derivative feedback, there exists a real matrix $\boldsymbol{F}$ if and only if the $(n+m) \times 1$-dimensional parameter vectors, $\boldsymbol{\psi}_{i j}^{k}$, are arbitrary chosen under the condition that the columns of matrix $\boldsymbol{V}$ are linearly independent.

Finally, a real gain matrix $\boldsymbol{F}$ is expressed as:

$$
\boldsymbol{F}=\boldsymbol{Z} \boldsymbol{V}^{-}
$$

where

$$
\boldsymbol{V}=\left[V_{1}\left(\lambda_{1}\right), \ldots, V_{s}\left(\lambda_{s}\right)\right] \text { and } \boldsymbol{Z}=\left[Z_{1}\left(\lambda_{1}\right), \ldots, Z_{s}\left(\lambda_{s}\right)\right] .
$$

Then the feedback gain matrix $\boldsymbol{F}$ is parameterized directly in terms of the eigenstructure of the closed-loop system.

There exists a real feedback gain matrix $\boldsymbol{F}$, such that (32) holds, if and only if the following three conditions are satisfied:

1. The assigned eigenvalues are symmetric with respect to the real axis.

2. The vectors $\left\{\mathbf{v}_{i j}^{k} \in \mathrm{C}^{n}, i=1, \ldots, s, j=1, \ldots, q_{i}, k=1, \ldots, p_{i j}\right\}$ are linearly independent and for complex-conjugate poles, $\lambda_{i_{1}}=\bar{\lambda}_{i_{2}}$ then $\mathbf{v}_{i_{1} j}^{k}=\overline{\mathbf{v}}_{i_{2} j}^{k}$.

3. There exists a set of vectors

$\left\{\mathbf{z}_{i j}^{k} \in \mathrm{C}^{m}, i=1, \ldots, s, j=1, \ldots, q_{i}, k=1, \ldots, p_{i j}\right\}$,

satisfying (32) and $\mathbf{z}_{i_{1} j}^{k}=\overline{\mathbf{z}}_{i_{2} j}^{k}$ for $\lambda_{i_{1}}=\bar{\lambda}_{i_{2}}$.

\section{Illustrative examples}

In this section, we present numerical examples to illustrate the feasibility and effectiveness of the proposed eigenstructure assignment technique.

\section{Example 1:}

Consider a single-input linear system described in the state-space form

$$
\dot{\boldsymbol{x}}(t)=\left(\begin{array}{ll}
1 & 2 \\
0 & 3
\end{array}\right) \boldsymbol{x}(t)+\left(\begin{array}{l}
0 \\
1
\end{array}\right) \boldsymbol{u}(t)
$$

In the following, we consider the assignment of two different cases:

Case 1: The desired closed-loop eigenvalues are selected as, $\{-3$ and -4$\}$.

For $\lambda_{1}=-3, \quad\left[\lambda_{1} \boldsymbol{I}_{n}-\boldsymbol{A}, \lambda_{1} \boldsymbol{B}\right] \xi_{1}=\left(\begin{array}{ccc}-4 & -2 & 0 \\ 0 & -6 & -3\end{array}\right) \boldsymbol{\xi}_{1}=\boldsymbol{0}$, arbitrarily selecting $\boldsymbol{\xi}_{1}=[-0.5,1,-2]^{\top}$ leads to $K\left(\begin{array}{c}-0.5 \\ 1\end{array}\right)=-2$.

For $\lambda_{2}=-4, \quad\left[\lambda_{2} \boldsymbol{I}_{n}-\boldsymbol{A}, \lambda_{2} \boldsymbol{B}\right] \boldsymbol{\xi}_{2}=\left(\begin{array}{ccc}-5 & -2 & 0 \\ 0 & -7 & -4\end{array}\right) \xi_{2}=\mathbf{0}$,

taking $\boldsymbol{\xi}_{2}=[-0.4,1,-7 / 4]^{\top}$

leads to $K\left(\begin{array}{c}-0.4 \\ 1\end{array}\right)=-7 / 4$.

Taking the two equations together, we can write

$$
\boldsymbol{K}\left(\begin{array}{cc}
-0.5 & -0.4 \\
1 & 1
\end{array}\right)=\left(\begin{array}{ll}
-2 & -7 / 4
\end{array}\right) .
$$

Finally, the unique state-derivative feedback gain matrix is

$$
\boldsymbol{K}=[2.5,0.75] \text {. }
$$

Case 2: The desired eigenvalues are, $\{-1$ and -1$\}$.

For $\lambda=-1, \quad\left[\lambda \boldsymbol{I}_{n}-\boldsymbol{A}, \lambda \boldsymbol{B}\right] \boldsymbol{\xi}_{11}^{1}=\left(\begin{array}{ccc}-2 & -2 & 0 \\ 0 & -4 & -1\end{array}\right) \boldsymbol{\xi}_{11}^{1}=\boldsymbol{0}$, leads to $\boldsymbol{\xi}_{11}^{1}=[-1,1,-4]^{\top}$ and $\boldsymbol{K}\left(\begin{array}{c}-1 \\ 1\end{array}\right)=-4$.

The generalized eigenvector equation is,

$$
\left[\lambda \boldsymbol{I}_{n}-\boldsymbol{A}, \lambda \boldsymbol{B}\right] \boldsymbol{\xi}_{12}^{1}=-\left[\boldsymbol{I}_{n}, \boldsymbol{B}\right] \boldsymbol{\xi}_{11}^{1},
$$

and, $\quad\left(\begin{array}{ccc}-2 & -2 & 0 \\ 0 & -4 & -1\end{array}\right) \boldsymbol{\xi}_{12}^{1}=\left(\begin{array}{ccc}-1 & 0 & 0 \\ 0 & -1 & -1\end{array}\right)\left(\begin{array}{c}-1 \\ 1 \\ -4\end{array}\right)=\left(\begin{array}{l}1 \\ 3\end{array}\right)$,

$$
\text { then } \xi_{12}^{1}=[-1.5,1,-7]^{\top} \text { and } \boldsymbol{K}\left(\begin{array}{c}
-1.5 \\
1
\end{array}\right)=-7 \text {. }
$$

And the gain equation can be written as

$$
\boldsymbol{K}\left(\begin{array}{cc}
-1 & -1.5 \\
1 & 1
\end{array}\right)=\left(\begin{array}{ll}
-4 & -7
\end{array}\right)
$$

Finally, the unique feedback gain is

$$
\boldsymbol{K}=[6,2] \text {. }
$$

\section{Example 2:}

Consider a multi-input controllable linear system

$$
\dot{x}(t)=\left(\begin{array}{ll}
1 & 2 \\
0 & 3
\end{array}\right) \boldsymbol{x}(t)+\left(\begin{array}{ll}
1 & 0 \\
0 & 1
\end{array}\right) \boldsymbol{u}(t)
$$

In the following, we consider the assignment of two different cases:

Case 1: The desired closed-loop eigenvalues are selected as $\{-3$ and -5$\}$.

For $\lambda_{1}=-3, \quad\left[\lambda_{1} \boldsymbol{I}_{n}-\boldsymbol{A}, \lambda_{1} \boldsymbol{B}\right] \xi_{1}=\left(\begin{array}{cccc}-4 & -2 & -3 & 0 \\ 0 & -6 & 0 & -3\end{array}\right) \xi_{1}=\mathbf{0}$,

taking $\mathbf{v}_{1}=[a, b]^{\top}$,

leads to $\boldsymbol{\xi}_{1}=[a, b,-4 a / 3-2 b / 3,-2 b]^{\top}$

and $\boldsymbol{K}\left(\begin{array}{l}a \\ b\end{array}\right)=\left(\begin{array}{c}-4 a / 3-2 b / 3 \\ -2 b\end{array}\right)$. 


$$
\begin{aligned}
\text { For } \lambda_{2}=-5, & {\left[\lambda_{2} \boldsymbol{I}_{n}-\boldsymbol{A}, \lambda_{2} \boldsymbol{B}\right] \boldsymbol{\xi}_{2}=\left(\begin{array}{cccc}
-6 & -2 & -5 & 0 \\
0 & -8 & 0 & -5
\end{array}\right) \boldsymbol{\xi}_{2}=\boldsymbol{0}, } \\
& \text { choosing } \mathbf{v}_{2}=[c, d]^{\top}, \\
& \text { then } \boldsymbol{\xi}_{2}=[c, d,-6 c / 5-2 d / 5,-8 d / 5]^{\top}, \\
& \text { and } \boldsymbol{K}\left(\begin{array}{l}
c \\
d
\end{array}\right)=\left(\begin{array}{c}
-6 c / 5-2 d / 5 \\
-8 d / 5
\end{array}\right) .
\end{aligned}
$$

Then, the feedback gain matrix equation is

$$
\boldsymbol{K}\left(\begin{array}{ll}
a & c \\
b & d
\end{array}\right)=\left(\begin{array}{cc}
-4 a / 3-2 b / 3 & -6 c / 5-2 d / 5 \\
-2 b & -8 d / 5
\end{array}\right) .
$$

Then any values of $a, b, c$ and $d$ will give a valid gain matrix as long as the required inverse exists. To give the orthogonal set of the closed-loop eigenvector, we set $a=d=1$ and $b=c=0$.

The gain matrix is

$$
\boldsymbol{K}=\left(\begin{array}{cc}
-4 / 3 & -2 / 5 \\
0 & -8 / 5
\end{array}\right)
$$

Case 2: The desired closed-loop poles are, $\{-1$ and -1$\}$.

$$
\begin{aligned}
\text { For } \lambda_{1}=-1, & {\left[\lambda \boldsymbol{I}_{n}-\boldsymbol{A}, \lambda \boldsymbol{B}\right] \boldsymbol{\xi}_{11}^{1}=\left(\begin{array}{cccc}
-2 & -2 & -1 & 0 \\
0 & -4 & 0 & -1
\end{array}\right) \boldsymbol{\xi}_{11}^{1}=\boldsymbol{0}, } \\
& \text { taking } \mathbf{v}_{11}^{1}=[a, b]^{\top}, \\
& \text { leads to } \boldsymbol{\xi}_{11}^{1}=[a, b,-2 a-2 b,-4 b]^{\top} .
\end{aligned}
$$

The generalized eigenvector equation is,

$$
\left[\lambda \boldsymbol{I}_{n}-\boldsymbol{A}, \lambda \boldsymbol{B}\right] \boldsymbol{\xi}_{12}^{1}=-\left[\boldsymbol{I}_{n}, \boldsymbol{B}\right] \boldsymbol{\xi}_{11}^{1}
$$

$\left(\begin{array}{cccc}-2 & -2 & -1 & 0 \\ 0 & -4 & 0 & -1\end{array}\right) \boldsymbol{\xi}_{12}^{1}=\left(\begin{array}{cccc}-1 & 0 & -1 & 0 \\ 0 & -1 & 0 & -1\end{array}\right) \boldsymbol{\xi}_{11}^{1}=\left(\begin{array}{c}a+2 b \\ 3 b\end{array}\right)$,

taking $\mathbf{v}_{12}^{1}=[c, d]^{\top}$, leads to

$\boldsymbol{\xi}_{12}^{1}=[c, d,-a-2 b-2 c-2 d,-3 b-4 d]^{\top}$.

Then, the gain matrix equation can be written as

$$
\boldsymbol{K}=\left(\begin{array}{ll}
a & c \\
b & d
\end{array}\right)=\left(\begin{array}{cc}
-2 a-2 b & -a-2 b-2 c-2 d \\
-4 b & -3 b-4 d
\end{array}\right) .
$$

We can set $a=d=1$ and $b=c=0$.

Then, the gain matrix is

$$
\boldsymbol{K}=\left(\begin{array}{cc}
-2 & -3 \\
0 & -4
\end{array}\right) \text {. }
$$

\section{Example 3:}

Consider a controllable and observable, multi-input linear system

$$
\begin{aligned}
\dot{x}(t) & =\left(\begin{array}{cc}
0 & 1 \\
-3 & -4
\end{array}\right) \boldsymbol{x}(t)+\left(\begin{array}{ll}
1 & 0 \\
0 & 1
\end{array}\right) \boldsymbol{u}(t), \\
y(t) & =\left(\begin{array}{ll}
1 & 1
\end{array}\right) \boldsymbol{x}(t)
\end{aligned}
$$

In the following we consider the first eigenvalue at $\left(\lambda_{1}=-5\right)$,

$\left[\lambda_{1} \boldsymbol{I}_{n}-\boldsymbol{A}, \lambda_{1} \boldsymbol{B}\right] \boldsymbol{\psi}_{1}=\left(\begin{array}{cccc}-5 & -1 & -5 & 0 \\ 3 & -1 & 0 & -5\end{array}\right) \boldsymbol{\psi}_{1}=\mathbf{0}$

taking $\mathbf{v}_{1}=[a, b]^{\top}$,

leads to $\psi_{1}=[a, b,-a-0.2 b, 0.6 a-0.2 b]^{\top}$.
This means $\boldsymbol{F C}\left(\begin{array}{l}a \\ b\end{array}\right)=\left(\begin{array}{c}-a-0.2 b \\ 0.6 a-0.2 b\end{array}\right)$.

Hence, $\quad \boldsymbol{F C}\left(\begin{array}{l}a \\ b\end{array}\right)=\boldsymbol{F}(a+b)=\left(\begin{array}{c}-a-0.2 b \\ 0.6 a-0.2 b\end{array}\right)$.

The gain matrix is

$$
\boldsymbol{F}=\frac{1}{a+b}\left(\begin{array}{c}
-a-0.2 b \\
0.6 a-0.2 b
\end{array}\right)
$$

Clearly indicating that the real output-derivative gain matrix is not unique and a finite number of valid choices for $a$ and $b$ are possible.

The closed-loop system equation can be computed as

$$
\begin{aligned}
& \left(\boldsymbol{I}_{n}+\boldsymbol{B F C}\right)^{-1} \boldsymbol{A}= \\
& \left(\boldsymbol{I}_{n}+\frac{1}{a+b}\left(\begin{array}{cc}
-a-0.2 b & -a-0.2 b \\
0.6 a-1.8 b & 0.6 a-0.2 b
\end{array}\right)\right)^{-1}\left(\begin{array}{cc}
0 & 1 \\
-3 & -4
\end{array}\right)= \\
& \frac{1}{a+b}\left(\begin{array}{cc}
-5 a-b & -4 b \\
-4 b & -a-5 b
\end{array}\right)
\end{aligned}
$$

then it is easily to shown that the closed-loop system has eigenvalues at -5 and -1 , regardless of the $a$ and $b$ values.

It has been shown, how the eigenstructure assignment approach can be used to design a controller-based state-derivative and partial output-derivative feedback control, which yields a closed-loop system with specified characteristics. The approach is relevant for design with preservation of stability when some necessary and sufficient conditions are provided. Compared to state feedback, the state-derivative feedback controller in some cases achieves the same performance with lower gain elements. From the practical point of view, it is desirable to determine feedback with small gains. Intuitively, this must be advantageous, since small gains lead to smaller control signals, and thus to less energy consumption.

\section{Conclusions}

This paper proposes a parametric approach for solving the eigenstructure assignment problem via state-derivative feedback. The necessary conditions to ensure solvability are that the system is controllable and the open-loop system matrix is nonsingular. The main result of this work is an efficient algorithm for solving the eigenstructure assignment problem of linear systems via state-derivative feedback. The extra degrees of freedom on the choice of feedback gains can be exploited to further to improve the closed-loop robustness against perturbation. Additionally, the eigenstructure assignment problem via partial output-derivative feedback is introduced. The numerical examples prove the feasibility and effectiveness of the proposed technique.

\section{References}

[1] Abdelaziz T. H. S., Valášek M.: "Pole placement for SISO linear systems by state-derivative feedback." (Submitted to IEE Proceeding Part D: Control Theory and Applications).

[2] Abdelaziz T. H. S., Valášek M.: "A direct algorithm for pole placement by state-derivative feedback for single- 
-input linear systems." Acta Polytechnica, Vol. 6 (2003), p.52-60.

[3] Abdelaziz T. H. S., Valášek M.: “A direct algorithm for pole placement by state-derivative feedback for multi-input linear systems." (to appear).

[4] Duan G. R: "Solution of the equation $A V+B W=V F$ and their application to eigenstructure assignment in linear systems." IEEE Transactions on Automatic Control, Vol. 38 (2) (1993), p. 276-280.

[5] Fahmy M. M., Tantawy H. S.: "Eigenstructure assignment via linear state feedback control." International Journal of Control, Vol. 40(1984), No. 1, p. 161-178.

[6] Fahmy M. M., O'Reilly J.: "Eigenstructure assignment in linear multivariable systems a parametric solution." IEEE Transactions on Automatic Control, Vol. 28 (1983), No. 10, p. 990-994.

[7] Fahmy M. M., O’Reilly J.: “On eigenstructure assignment in linear multivariable systems." IEEE Transactions on Automatic Control, Vol. 27 (1982), No. 3, p. 690-693.

[8] Brogan W. L.: Modern control theory, Englewood Cliffs, NJ: Prentice-Hall, Inc., 1991.

[9] Lewis F. L.: Applied Optimal Control and Estimation, Digital Design and Implementation. Prentice-Hall and Texas Instruments, Englewood Cliffs, NJ., 1992.

[10] Preumont A., Loix N., Malaise D., Lecrenier O.: "Active Damping of Optical Test Benches with Acceleration Feedback." Machine Vibration, Vol. 2, (1993), p. 119-124.

[11] Preumont A.: Vibration Control of Active Structures, Kluwer, 1998.

[12] Bayon de Noyer M. P., Hanagud S. V.: "Single Actuator And Multi-Mode Acceleration Feedback Control." Adaptive Structures and Material Systems, ASME, AD, Vol. 54 (1997), p. 227-235.

[13] Bayon de Noyer M. P., Hanagud S. V.: A Comparison of H2 Optimized Design and Cross-Over Point Design For
Acceleration Feedback Control. Proceedings of $39^{\text {th }}$ AIAA/ASME/ ASCE/AHS, Structures, Structural Dynamics and Materials Conference, Vol. 4, 3250-3258, (AIAA-98-2091), 1998.

[14] Olgac N., Elmali H., Hosek M., Renzulli M.: “Active Vibration Control of Distributed Systems Using Delayed Resonator with Acceleration Feedback." Transactions of ASME Journal of Dynamic Systems, Measurement and Control, Vol. 119 (1997), p. 380.

[15] Kejval J., Sika Z., Valášek M.: Active Vibration Suppression of a Machine, Proceedings of Interaction and Feedbacks 2000, UT AV CR, Praha, 2000, p. 75-80.

[16] Deur J., Peric N.: A Comparative Study of Servosystems with Acceleration Feedback. Proceedings of the $35^{\text {th }}$ IEEE Industry Applications Conference, Roma, Italy, Vol. 2 (2000), p. 1533-1540.

[17] Ellis G.: Cures for Mechanical Resonance in Industrial Servo Systems. Proceedings of PCIM 2001 Conference, Nuremberg, 2001.

[18] Horn R. A., Johnson C. R.: Matrix Analysis. Cambridge University Press, Cambridge 1986.

Eng. Taha H. S. Abdelaziz, Ph.D.

e-mail: tahahelmy@yahoo.com

Doc. Michael Valášek, DrSc.

e-mail:valasek@fsik.cvut.cz

Department of Mechanics

Czech Technical University in Prague

Faculty of Mechanical Engineering

Karlovo nám. 13

12135 Praha 2, Czech Republic 